\title{
O uso das tecnologias digitais na infância pode influenciar nos modos de interação social? Evidências de uma revisão sistemática de literatura
}

\author{
Can the use of digital technologies in childhood influence the \\ modes of social interaction? Evidences of a systematic literature \\ review
}

\author{
Daniela Karine Ramos ${ }^{1}$ \\ Ana Paula Knaul2
}

\section{Resumo}

A influência que as tecnologias digitais têm gerado na qualidade das relações humanas torna-se cada vez mais relevante de ser observada devido a sua onipresença nas práticas culturais. Ao mesmo tempo reconhece-se o papel fundamental das interações sociais para o processo de desenvolvimento humano. Diante disso, este trabalho tem como objetivo analisar pesquisas que abordam a repercussão do acesso às tecnologias digitais sobre os modos de interação social na infância, por meio da realização de uma revisão sistemática. A revisão foi conduzida nas bases de dados ERIC, Science Direct, BDTD IBICT, observando duas etapas. A busca inicial resultou em 475 trabalhos, então se procedeu à análise dos títulos e resumos com base nos critérios de inclusão, o que resultou na triagem de oito artigos que foram analisados na íntegra. De modo geral, pôde-se observar nos desfechos descritos que os trabalhos sugerem que o uso das tecnologias digitais não limita ou substitui as interações sociais e revelam que as mesmas podem ser usadas para motivarem as relações sociais, ampliando as formas de

\footnotetext{
${ }^{1}$ Doutora em Educação, Professora do Programa de Pós-Graduação em Educação e do Departamento de Metodologia de Ensino da Universidade Federal de Santa Catarina ${ }^{2}$ Doutoranda em Educação na Linha de Pesquisa Educação e Comunicação da Universidade Federal de Santa Catarina (PPGE/UFSC) Interfaces da Educ., Paranaíba, v.11, n.32, p. 159 - 187, 2020
} 
comunicação e contribuindo com o desenvolvimento de competências digitais.

Palavras-chave: Habilidades sociais. Mediação. Família. Escola.

\section{Abstract}

The influence that digital technologies have generated on the quality of human relations becomes increasingly relevant to be observed due to their ubiquity in cultural practices. At the same time, the fundamental role of social interactions for the process of human development is recognized. Therefore, this paper aims to analyze research that addresses the impact of access to digital technologies on modes of social interaction in childhood, through a systematic review. The review was conducted in the ERIC, Science Direct, BDTD IBICT databases, observing two steps. The initial search resulted in 475 papers, then titles and abstracts were analyzed based on the inclusion criteria, resulting in the screening of eight articles that were fully analyzed. In general, it can be observed in the described outcomes that the works suggest that the use of digital technologies does not limit or replace social interactions and reveal that they can be used more to motivate social relations, broadening the forms of communication and contributing with the development of digital competences

Therefore, this paper aims to analyze research that addresses the impact of access to digital technologies on modes of social interaction in childhood, through a systematic review. The review was conducted in the Eric, Science Direct, Bdtd Ibict databases, observing two steps. The initial search found in 475 papers, then titles and abstracts were analyzed based on the inclusion criteria, resulting in the screening of eight articles that were analyzed in full. In general, it can be observed in the described outcomes that the works suggest that digital technologies can be used more to motivate social relations, expanding the forms of communication and the development of digital competences, rather than limiting or disabling interactions. 
Keywords: Social Skills. Mediation. Family. School

\section{Introdução}

No sentido de compreender e problematizar a influência que as tecnologias digitais têm gerado na qualidade das relações humanas, pesquisas em diferentes áreas do conhecimento têm possibilitado ampliar a gama de reflexões teóricas e empíricas a respeito desse campo ainda muito recente na contemporaneidade (UHLS et al., 2014; TSCHOLL; LINDGREN, 2014; SANTISARUN; BOONKRONG, 2015). Essa temática torna-se cada vez mais relevante devido à onipresença das tecnologias digitais nas práticas culturais de muitas crianças e adolescentes, revelando-se fortemente integradas aos modos de se relacionar e conviver em comunidade.

Atualmente, é comum encontrar pessoas utilizando uma tecnologia digital, um smartphone, por exemplo, em detrimento da interação presencial com pessoas próximas, sendo esta uma escolha e opção no momento. No entanto, na medida em que isso ganha proporção de tempo maior do que o envolvimento em outras atividades é que se faz necessária uma atenção às influências do que esse hábito pode gerar em longo prazo, especialmente na infância em que as relações sociais são fundamentais ao desenvolvimento já que se pode entender que "todas as funções superiores originam-se das relações reais entre indivíduos humanos" (VYGOTSKY, 2007, p. 58).

Diante do exposto, justifica-se a importância do presente trabalho no sentido de verificar se a presença das tecnologias digitais no cotidiano das crianças, ao invés de gerar experiências e conectá-las em suas atividades e na ampliação de seus repertórios, estão isolando-as umas das outras para atuação nas suas funcionalidades, em detrimento da relação com o outro quando estão presentes em um mesmo espaço físico. Assim, define-se como objetivo do estudo buscar evidências científicas sobre as influências do uso das tecnologias digitais sobre as relações sociais entre as crianças.

Com o aumento do uso do celular, conforme pode ser visualizado no Gráfico 1 a seguir, passando de 21\% em 2012 para 93\% em 2017 por 
crianças e adolescentes de 9 a 17 anos, o olhar para a transformação na forma como as relações sociais se estabelecem quando estão presencialmente uns com outros se faz necessária, para buscar evidências relacionadas as influências do mundo digital na qualidade e na forma como se estabelecem e se mantém essas relações sociais.

Gráfico 1: Crianças e adolescentes de 9 a 17 anos, por dispositivos utilizados para acessar a internet $(2012-2017)$.

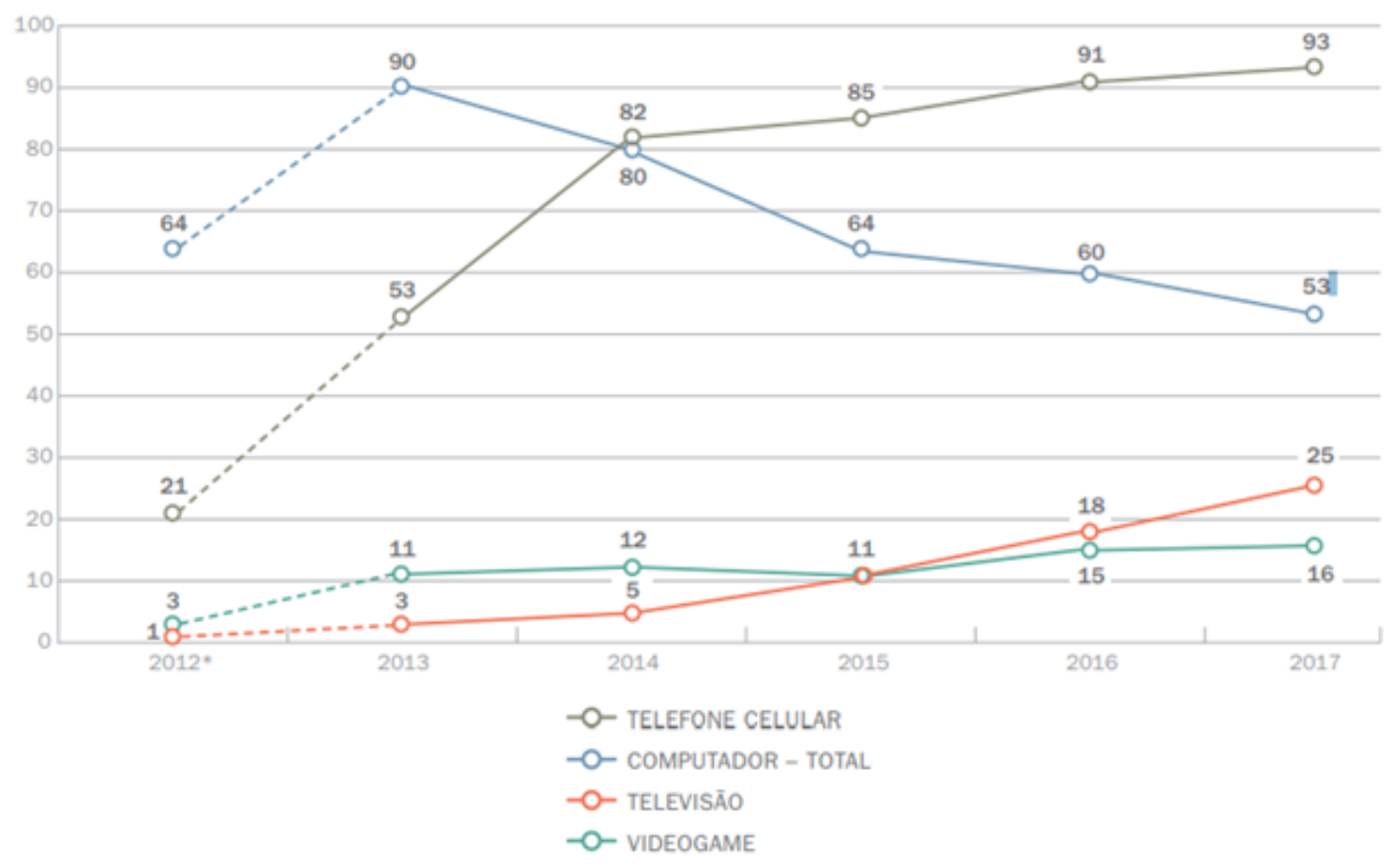

Fonte: TIC KIDS ONLINE BRASIL, 2018, p. 126.

$\mathrm{Na}$ pesquisa realizada por Uhls et al. (2014) que objetivou analisar se, a eliminação do uso das mídias digitais e o aumento da possibilidade de interação face a face durante cinco dias, entre 51 pré-adolescentes entre 11 e 13 anos, influenciaria numa melhora do reconhecimento não-verbal de emoções entre eles. Os resultados mostraram que combinadas a ausência da tecnologia e o aumento da interação face a face, melhoraram 
expressivamente a compreensão da emoção não verbal entre os pesquisados, quando comparado a um grupo controle.

Nas interações sociais face a face, a compreensão dos aspectos não verbais possibilita modificar o comportamento em resposta à reação do outro (KNAPP; HALL, 2010). Nesse sentido, caso essa compreensão seja limitada, as relações sociais podem ser prejudicadas devido à dificuldade de se entender as mensagens não verbais manifestadas, que podem dizer muito mais que a própria verbalização.

Assim, na comunicação mediada pelo digital, muitas vezes pautada em texto, gera a diminuição do contato face a face o que pode ter impactos sobre o desenvolvimento de habilidades sociais importantes para compreensão emocional nas relações sociais (GIEDD, 2012).

As habilidades sociais envolvem comportamentos verbais e não verbais, referindo-se a um conjunto de "comportamentos que ocorrem dentro do contexto interpessoal, que têm como finalidade comunicar com precisão emoções, sentimentos, opiniões, atitudes, direitos e necessidades pessoais" (ROCCA et al., 2010, p. 183).

Essas habilidades estão relacionadas à competência social que segundo Topper (2017) é uma capacidade de envolvimento para criar amizade e ser aceito pelos pares nas interações sociais. Assim, ampliam a compreensão de mundo social e dos diferentes pontos de vista das pessoas nas situações vividas (BOSACKI, ASTINGTON, 1999; ASTINGTON, 2003), ao mesmo tempo em que se relacionam coma eficiência de comunicação para uma ampla compreensão na relação entre pares (RUBIN, ROSE-KRASNOR, 1992) e como habilidade de correlacionar de forma positiva a regulação emocional, o bem-estar psicológico, o desempenho acadêmico e o sucesso num futuro trabalho (BLANDON et al., 2010).

Desse modo, percebe-se a complexidade de analisar comportamentos gerados a partir das relações sociais e entre elas, o que torna ainda mais desafiante a pesquisa sobre os possiveis impactos das tecnologias sobre as interações sociais na infância. De qualquer modo, a Academia Americana de 
Pediatria tem orientado os pais a seguirem uma abordagem colaborativa de aconselhamento junto às crianças, no sentido de ampliar seus olhares sobre os riscos online (BROWN, SHIFRIN, HILL, 2015). Segundo pesquisas realizadas, os riscos online às crianças referem-se a visualizar e postar conteúdo impróprio (LIVINGSTONE, 2014), enviar e receber mensagens ofensivas (LIVINGSTONE et al., 2013), publicar informações privadas em redes sociais (SANTISARUN; BOONKRONG, 2015) e confiar em informações da Internet sem verificação de outras fontes (EY; CUPIT, 2011).

Para seguir na reflexão sobre a qualidade das interações sociais mediante o uso da tecnologia digital, na pesquisa realizada por Tscholl e Lindgren (2014) foi analisado o uso de uma tecnologia projetada para promover a aprendizagem por meio da interação das crianças com as suas famílias em um museu. Nessa proposta havia um simulador de realidade aumentada que exigia que as crianças acertassem um alvo localizado atrás de um planeta para identificar a compreensão sobre gravidade na duplicação da velocidade de um asteroide, enquanto as famílias tinham acesso a outros conteúdos para questionar as crianças a respeito da atividade realizada na simulação.

De acordo com Tscholl e Lindgren (2014), foi a partir da motivação para a interação entre as crianças e suas famílias que a aprendizagem foi possibilitada na mediação com o simulador. Como a experiência móvel exigiu movimentos físicos das crianças para o alcance do objetivo proposto, quando aliada a mediação das famílias, tornou possivel a elas compreenderem a informação e conteúdo apresentados, transformando a qualidade e o nível da experiência.

As tecnologias apresentam-se como um universo de possibilidades de artefatos, usos e modos de interação e acesso as informações. Assim, qualquer pesquisa que se proponha a analisar impactos precisa considerar os modos de ação e operação sobre essas tecnologias, o que torna inviável se chegar a generalizações e certezas. As tecnologias digitais envolvem uma cultura de uso das mídias permeada pela configuração social que criam 
novos modos de pensar, comunicar, criar, produzir, trabalhar, aprender e viver (COLL; MONEREO, 2010; ALMEIDA; SILVA, 2011).

Diante disso, este trabalho se propõe a levantar e analisar pesquisas que abordem as interferências do acesso às tecnologias digitais sobre os modos de interação social na infância, por meio da realização de uma revisão sistemática, e assim verificar se esses usos em meios as relações sociais presenciais aproximam e contribuem para as relações, ou prejudicam e causam isolamento social no mesmo espaço e tempo.

\section{Metodologia}

De acordo com Sampaio e Mancini (2007), a revisão sistemática ao utilizar como fonte de dados às bibliografias produzidas relacionadas a um tema oferece "evidências relacionadas a uma estratégia de intervenção específica, mediante a aplicação de métodos explícitos e sistematizados de busca, apreciação crítica e sintese da informação selecionada" (p. 84).

Considerando a especificidade do tema e a escassez de trabalhos na área no Brasil, optou-se para fazer a busca dos últimos 10 anos, que compreendeu o período de 2009 a 2018, em três bases de dados internacionais, que centralizam uma grande quantidade de produções, sendo elas: Educational Resources Information Centre (ERIC), Science Direct, BDTD IBICT. A escolha por essas bases de dados se deu em função das áreas de conhecimentos e tipo de documentos integrados. A ERIC refere-se a uma base na área de educação, a Science Direct é uma base mais ampla e integra diversas áreas do conhecimento e a BDTD IBICT integra informações sobre teses e dissertações do Brasil. .

A realização da revisão observou os passos descritos por Okoli (2015) para revisão sistemática, incluindo os seguintes procedimentos: a) a delimitação do objetivo da revisão; b) a elaboração do protocolo para sua condução, que consistiu numa planilha digital onde foram organizados todos os trabalhos encontrados e os seus respectivos dados, considerando os 
critérios de inclusão e filtragem e, c) a realização da triagem pautada nos critérios de inclusão c e filtragem.

A primeira busca utilizando a combinação de termos definidos e os filtros referentes a língua, período de tempo e a presença dos termos no título ou resumo resultou em 475 artigos.

Quadro 1. Filtros e resultados da primeira busca.

\begin{tabular}{|c|c|c|c|}
\hline Termos & Base & Filtros & Resultados \\
\hline \multirow{3}{*}{$\begin{array}{l}\text { child OR children, AND } \\
\text { social relationships OR } \\
\text { social interaction AND } \\
\text { digital technologies OR } \\
\text { mobile technology OR } \\
\text { mobile Technologies }\end{array}$} & ERIC & $\begin{array}{c}\text { Inglês, espanhol e } \\
\text { português; } 2009 \text { a } \\
2018 \text {; termos presentes } \\
\text { no título ou resumo. }\end{array}$ & 72 \\
\hline & $\begin{array}{l}\text { Science } \\
\text { Direct }\end{array}$ & $\begin{array}{c}\text { Inglês, espanhol e } \\
\text { português; } 2009 \text { a } \\
2018 \text {; termos presentes } \\
\text { no título ou resumo. } \\
\text { Tipo: Review articles e } \\
\text { Research articles }\end{array}$ & 323 \\
\hline & Bdtd Ibict & $\begin{array}{c}\text { Inglês, espanhol e } \\
\text { português; } 2009 \text { a } \\
2018 \text {; termos presentes } \\
\text { no título ou resumo. }\end{array}$ & 80 \\
\hline
\end{tabular}

Fonte: Elaboração da autora, 2018.

A seguir procedeu-se a triagem considerando os critérios de inclusão definidos: pesquisa tendo como participantes crianças com até 12 anos de idade, a descrição e análise do uso de tecnologias digitais, a realização de pesquisa empírica e o desfecho relacionado à influência das tecnologias digitais sobre a interação social.

Em todo processo foram realizados os registros e a descrição dos procedimentos. Na segunda etapa procedeu-se a extração dos dados e as informações aplicáveis dos estudos incluídos na revisão, observando os critérios de inclusão aplicados na leitura do resumo e texto na integra, tendo em vista a avaliação da qualidade na triagem para exclusão de documentos por qualidade insuficiente (OKOLI, 2015). Assim, de 475 pesquisas encontradas, somente sete trabalhos da ERIC e um trabalho da BDTD IBICT apresentaram aproximações com a temática desta pesquisa, atendendo aos critérios de inclusão. 
A partir disso, conduziu-se o procedimento de síntese e análise dos estudos de oito trabalhos, destacando-se especialmente os objetivos, a metodologia utilizada e os resultados descritos. A perspectiva seguida na análise dos trabalhos foi de elucidar e destacar os pontos abordados em cada uma das pesquisas, sem fazer escolhas teóricas ou destacar preferências na forma de análise dos trabalhos, pois se considera fundamental em relação ao objetivo dessa revisão sistemática não partir de pré-concepções ou hipóteses sobre os possiveis resultados. A intenção foi justamente conhecer diferentes perspectivas teóricas e metodológicas na análise do fenômeno em questão. Em alguns momentos, no decorrer das discussões foram sendo apresentados pontos que merecem uma maior atenção em relação às pesquisas na temática em questão, o que em momento algum deslegitima ou diminui o que foi encontrado nesta revisão sistemática.

\section{Resultados}

As buscas realizadas nos últimos 10 anos revelaram que o tema ainda é muito recente, pois os trabalhos que foram encontrados referem-se aos últimos cinco anos, conforme pode ser visualizado no Gráfico 1, apresentando um número maior de pesquisas realizadas no ano de 2017.

Gráfico 1. Relação dos trabalhos encontrados nos últimos 10 anos.

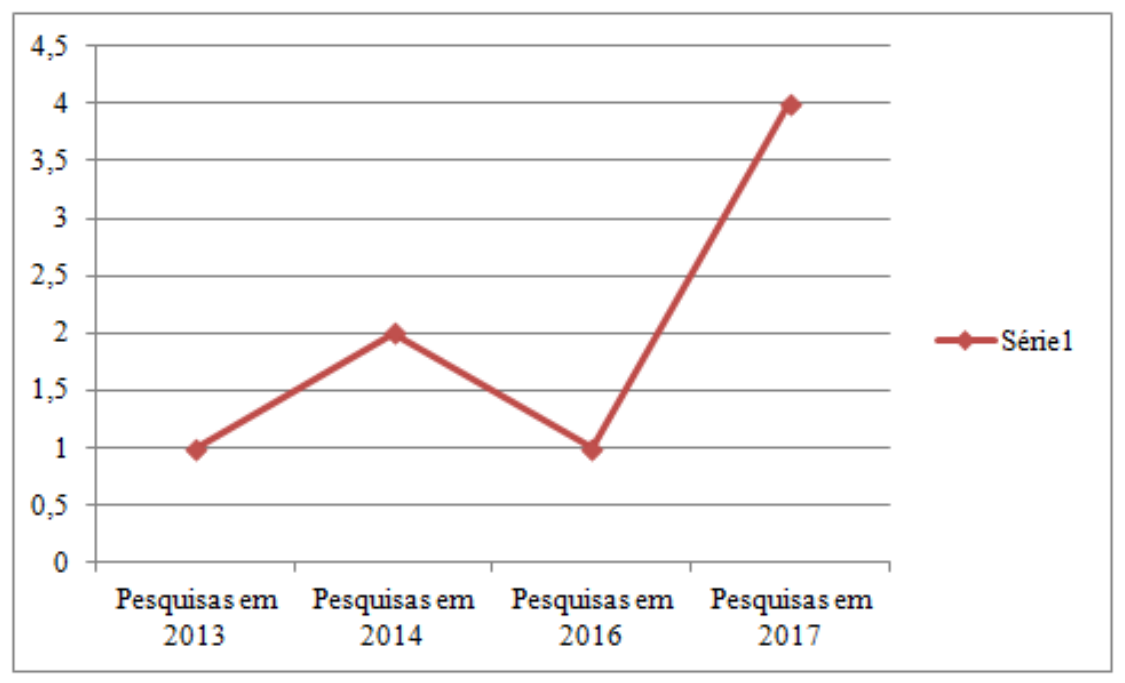

Interfaces da Educ., Paranaíba, v.11, n.32, p. 159 - 187, 2020 
Fonte: Elaboração da autora, 2018.

Destes oito trabalhos encontrados, quatro deles foram realizados nos Estados Unidos, três no Reino Unido e apenas um teve origem no Brasil, o que demonstra um déficit de pesquisas nessa área na produção científica internacional e nacional.

Para detalhar um pouco mais sobre os resultados obtidos com a revisão sistemática, destaca-se a seguir as amostras analisadas e as metodologias propostas nos trabalhos encontrados que levaram aos dados apresentados.

Quadro 3. Amostra e metodologia utilizada nos trabalhos encontrados na revisão sistemática.

\begin{tabular}{|c|c|c|}
\hline $\begin{array}{c}\text { ID / TÍTULO / } \\
\text { AUTOR/ BASE DE } \\
\text { DADOS }\end{array}$ & AMOSTRA & METODOLOGIA \\
\hline $\begin{array}{l}\text { 1. Parent-Child } \\
\text { Moments of Meeting } \\
\text { in Art-Making with } \\
\text { Collage, iPad, } \\
\text { Tuxpaint and Crayons } \\
\text { Autores: Mona Sakr e } \\
\text { Natalia Kucirkova } \\
\text { Base de Dados: ERIC }\end{array}$ & $\begin{array}{l}1 \text { criança de } \\
\text { três anos e o } \\
\text { pai. }\end{array}$ & $\begin{array}{l}\text { Auto-relato do pai com } 8 \\
\text { observações de } 10 \text { a } 30 \\
\text { minutos. } \\
\text { Utilização de recursos: lápis de } \\
\text { cor e papel branco; colagem em } \\
\text { papel colorido; o software de } \\
\text { criação de arte digital tuxpaint } \\
\text { em um laptop e a aplicação } \\
\text { digital Nossa História no iPad. } \\
\text { Gravação em vídeo de cada } \\
\text { episódio/observação com uma } \\
\text { câmera montada em um tripé } \\
\text { ou através de uma câmera de } \\
\text { mão quando os movimentos dos } \\
\text { participantes faziam isso }\end{array}$ \\
\hline
\end{tabular}




\begin{tabular}{|c|c|c|}
\hline & & necessário. \\
\hline $\begin{array}{l}\text { 2. An Action Research } \\
\text { Study Investigating } \\
\text { Children's Use of an } \\
\text { iPad during Free Play } \\
\text { in a Kindergarten } \\
\text { Classroom: An } \\
\text { Exploration of } \\
\text { Teaching Pedagogy } \\
\text { and Children's } \\
\text { Learning, Social } \\
\text { Interactions, and } \\
\text { Digital Literacy } \\
\text { Autor: Tara Reynolds- } \\
\text { Blankenship } \\
\text { Base de Dados: ERIC }\end{array}$ & $\begin{array}{l}1 \text { professor e } \\
25 \text { crianças } \\
\text { da pré-escola }\end{array}$ & $\begin{array}{l}\text { Diário reflexivo de pesquisador } \\
\text { durante o periodo de } 3 \text { meses. } \\
\text { Dados qualitativos consistindo } \\
\text { de notas de campo, } \\
\text { observações, gravações de } \\
\text { áudio, entrevistas, realizando } \\
\text { uma análise comparativa } \\
\text { constante para examinar os } \\
\text { dados de semelhanças e } \\
\text { diferenças. }\end{array}$ \\
\hline $\begin{array}{l}\text { 3. Meaning Making } \\
\text { with na iPad: A Case } \\
\text { Study of One Child's } \\
\text { Engagement with iPad } \\
\text { Applications within } \\
\text { Her Family Activity } \\
\text { System } \\
\text { Autor: Barbara M }\end{array}$ & $\begin{array}{l}1 \text { criança de } 5 \\
\text { anos de idade } \\
\text { e seus pais }\end{array}$ & $\begin{array}{l}\text { Estudo de caso com observação- } \\
\text { participante: notas de campo, } \\
\text { vídeos de interações, vídeos da } \\
\text { tela do iPad e notas de } \\
\text { entrevistas informais com } \\
\text { minha filha e meu marido. }\end{array}$ \\
\hline
\end{tabular}




\begin{tabular}{|c|c|c|}
\hline $\begin{array}{l}\text { Vokatis } \\
\text { Base de Dados: ERIC }\end{array}$ & & \\
\hline $\begin{array}{l}\text { 4. Preschool Children } \\
\text { and iPads: } \\
\text { Observations of Social } \\
\text { Interactions during } \\
\text { Digital Play } \\
\text { Autor: Sandra M. } \\
\text { Lawrence } \\
\text { Base de Dados: ERIC }\end{array}$ & $\begin{array}{l}20 \text { crianças } \\
\text { de } 5 \text { anos } \\
\text { numa pré- } \\
\text { escola }\end{array}$ & $\begin{array}{l}\text { O pesquisador reuniu-se com } \\
\text { cada criança individualmente } \\
\text { durante um periodo de } 8 \\
\text { semanas para analisar os usos } \\
\text { de } 6 \text { aplicativos educacionais } \\
\text { pelas crianças. Em horários } \\
\text { específicos durante o dia, os } \\
\text { pares de crianças tinham a } \\
\text { oportunidade de jogar com um } \\
\text { iPad compartilhado no centro de } \\
\text { uma mesa durante } 15 \text { minutos. } \\
\text { No total ocorreram } 20 \text { sessões. } \\
\text { Entrevista semi-estruturada } \\
\text { com } 3 \text { professores. }\end{array}$ \\
\hline $\begin{array}{l}\text { 5. Tablets for two: } \\
\text { How dual tablets can } \\
\text { facilitate other- } \\
\text { wareness and } \\
\text { communication in } \\
\text { learning disabled } \\
\text { children with autism } \\
\text { Autores: Samantha } \\
\text { Holt NicolaYuill } \\
\text { Base de Dados: ERIC }\end{array}$ & $\begin{array}{l}8 \text { meninos com } \\
\text { idades entre } 5 \\
\text { e } 12 \text { anos com } \\
\text { diagnóstico de } \\
\text { autismo e } \\
\text { incapacidade } \\
\text { de } \\
\text { aprendizagem } \\
\text { grave, de uma } \\
\text { escola de } \\
\text { Educação } \\
\text { Especial }\end{array}$ & $\begin{array}{l}\text { O estudo foi realizado em uma } \\
\text { sala separada, próxima às salas } \\
\text { de aula das crianças, utilizando } \\
\text { um tablet compartilhado com } \\
\text { mouses com controle duplo. }\end{array}$ \\
\hline $\begin{array}{l}\text { 6. Parental Perception } \\
\text { of Mobile Device }\end{array}$ & $\begin{array}{l}401 \text { pais de } \\
\text { crianças de } 5 \text { a }\end{array}$ & $\begin{array}{l}\text { Aplicação de Questionário } \\
\text { online. Análise estatística }\end{array}$ \\
\hline
\end{tabular}




\begin{tabular}{|c|c|c|}
\hline $\begin{array}{l}\text { Usage in Children and } \\
\text { Social Competency } \\
\text { Autor: Christin } \\
\text { Topper } \\
\text { Base de Dados: ERIC }\end{array}$ & $\begin{array}{l}12 \text { anos que } \\
\text { possuem seus } \\
\text { próprios } \\
\text { dispositivos } \\
\text { móveis } \\
\text { pessoais }\end{array}$ & (Software $G *$ Power). \\
\hline $\begin{array}{l}\text { 7. O brincar na } \\
\text { educação infantil: a } \\
\text { influência das } \\
\text { tecnologias digitais } \\
\text { móveis no contexto da } \\
\text { brincadeira } \\
\text { Autor: Lorivane A. } \\
\text { Meneguzzo } \\
\text { Base de Dados: Bdtd } \\
\text { Ibcit }\end{array}$ & $\begin{array}{l}14 \text { crianças } \\
\text { de } 3 \text { e } 4 \text { anos } \\
\text { de idade e } 2 \\
\text { professores de } \\
\text { uma escola } \\
\text { municipal de } \\
\text { Educação } \\
\text { Infantil }\end{array}$ & $\begin{array}{l}\text { Pesquisa qualitativa. Para } \\
\text { desenvolver os dados, escolheu- } \\
\text { se a gravação em vídeo, que foi } \\
\text { feita em sala de aula onde as } \\
\text { crianças desenvolvem seus } \\
\text { jogos e recriações, todos os dias, } \\
\text { de manhã e à tarde. Os } \\
\text { dispositivos móveis - } \\
\text { smartphones e tablets - foram } \\
\text { inseridos entre os brinquedos e } \\
\text { outros itens da sala de aula, } \\
\text { estando disponiveis para as } \\
\text { crianças. }\end{array}$ \\
\hline $\begin{array}{l}\text { 8. An ecological } \\
\text { exploration of young } \\
\text { children's digital play: } \\
\text { framing children's } \\
\text { social experiences } \\
\text { with technologies in } \\
\text { early childhood } \\
\text { Autor: Lorna Arnott } \\
\text { Base de Dados: ERIC }\end{array}$ & $\begin{array}{l}90 \text { crianças de } \\
3 \text { a } 5 \text { anos de } \\
\text { duas pré- } \\
\text { escolas }\end{array}$ & $\begin{array}{l}\text { Utilizando um ciclo sistemático } \\
\text { e iterativo de coleta e análise de } \\
\text { dados, as interações das } \\
\text { crianças com } 24 \text { recursos } \\
\text { tecnológicos foram examinadas } \\
\text { durante um periodo de nove } \\
\text { meses e em três fases. Os dados } \\
\text { foram coletados através de } \\
\text { observações sistemáticas, } \\
\text { mapeamento de cluster }\end{array}$ \\
\hline
\end{tabular}




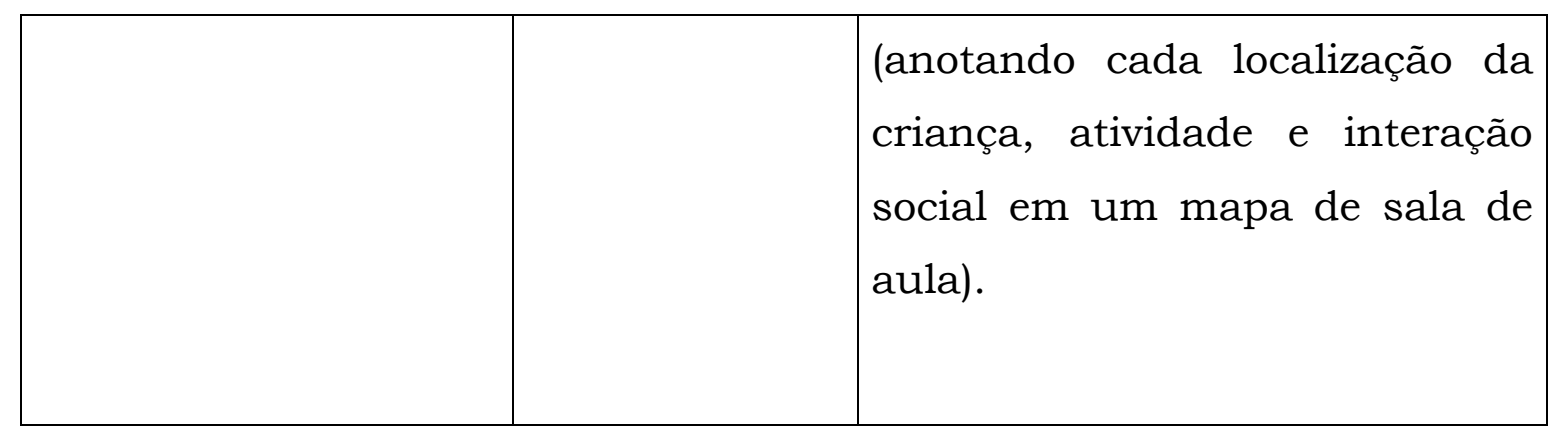

Fonte: Elaboração da autora, 2018.

Como pode ser observado, a maioria das pesquisas conduziu estudos de cunho experimental, em que $50 \%$ foram realizadas no espaço da préescola, apenas uma em uma escola de educação especial (HOLT; YUILL, 2017) e três no espaço domiciliar (VOKATIS, 2014; SAKR, KUCIRKOVA, 2017; TOPPER, 2017), sendo que uma desta última mencionada foi por meio de um questionário online enviado às famílias (TOPPER, 2017).

Dos 8 trabalhos encontrados, seis deles tiveram como público alvo crianças menores de cinco anos e somente duas pesquisas deram enfoque a crianças de 5 a 12 anos de idade (HOLT, YUILL, 2017; TOPPER, 2017) o que demonstra poucas pesquisas com enfoque em crianças de 6 a 12 anos.

Das duas pesquisas que analisaram a amostra que compreende a faixa etária de 5 a 12 anos que compreende crianças em idade de frequentar os anos iniciais do Ensino Fundamental, uma delas resumiu-se na aplicação de um questionário às famílias online (TOPPER, 2017) e a outra o enfoque foi em observações a partir do uso de uma tecnologia específica (HOLT, YUILL, 2017), oferecendo, com isso, poucos instrumentos de coleta de dados para triangulação na análise, que demonstraria maior relevância e até mesmo fidedignidade do dado obtido.

Além disso, a maioria das pesquisas não descrevia com detalhes e clareza informações sobre a forma, o tempo e a quantidade de aplicações com que os instrumentos metodológicos foram propostos, deixando dúvidas sobre quais aspectos foram determinantes na análise dos resultados em questão. 
Outro aspecto analisados nos trabalhos partir da sistematização das tecnologias digitais utilizadas nas pesquisas e o desfecho desse uso, tendo como enfoque a sua influência sobre a interação social.

Quadro 4. Tecnologias digitais utilizadas e o desfecho das pesquisas encontradas na revisão sistemática.

\begin{tabular}{|c|c|c|}
\hline $\begin{array}{c}\text { TECNOLOGIA } \\
\text { DIGITAL }\end{array}$ & ID & DESFECHO \\
\hline \multirow{2}{*}{ Tablet } & 1 & $\begin{array}{l}\text { As autoras identificaram que não foi a diferença da } \\
\text { tecnologia ser digital ou não que interferiu no nível de } \\
\text { interação entre o pai e a criança, mas foram as } \\
\text { potencialidades do que os recursos podiam fazer } \\
\text { fisicamente e como cada atividade era percebida e } \\
\text { dominada pelos dois, que determinou uma maior ou } \\
\text { menor proximidade entre ambos durante o uso. Nesse } \\
\text { caso, a tecnologia foi utilizada como um potencializador } \\
\text { da relação social entre pai e filho por meio de uma } \\
\text { estratégia de produção de arte colaborativa. }\end{array}$ \\
\hline & 2 & $\begin{array}{l}\text { Os resultados revelaram que a atividade mediada pelo } \\
\text { tablet cultivava um senso de comunidade e uma } \\
\text { transformação do ensino e da aprendizagem. A inclusão } \\
\text { do tablet deu início a um sistema de regras centralizado } \\
\text { na criança, que permitia às crianças mediar as } \\
\text { interações sociais entre os grupos e, como resultado, as } \\
\text { práticas de ensino foram alteradas. O papel do } \\
\text { professor foi impactado à medida que o professor cedeu } \\
\text { o controle, permitindo que as crianças medissem } \\
\text { conflitos e participassem ativamente de sua } \\
\text { aprendizagem. As ações foram mediadas pelo tablet e }\end{array}$ \\
\hline
\end{tabular}




\begin{tabular}{|c|c|c|}
\hline & & $\begin{array}{l}\text { permitiram que as crianças tomassem decisões e } \\
\text { colaborassem umas com as outras. }\end{array}$ \\
\hline & 3 & $\begin{array}{l}\text { Constatou-se que quando a ferramenta é adquirida } \\
\text { recentemente as interações dialógicas entre pais e a } \\
\text { criança são ainda mais dificeis de serem realizadas } \\
\text { devido a novidade que acarreta certo nível de imersão } \\
\text { da criança na tecnologia. Assim como o ambiente em si } \\
\text { também proporciona diferentes contextos de } \\
\text { apropriação. }\end{array}$ \\
\hline & 4 & $\begin{array}{l}\text { Revelaram que as crianças exibiram uma série de } \\
\text { comportamentos sociais, de competitivos a } \\
\text { colaborativos, enquanto se engajavam em quatro tipos } \\
\text { de brincadeiras digitais: prática / tarefa, exploração, } \\
\text { construção e simulação. As formas de brincar e os } \\
\text { comportamentos exibidos foram moldados por fatores } \\
\text { inter-relacionados das características e relações } \\
\text { individuais das crianças, cultura e rotinas de sala de } \\
\text { aula, visões e ações dos adultos, bem como aspectos do } \\
\text { próprio dispositivo digital. }\end{array}$ \\
\hline & 5 & $\begin{array}{l}\text { Os tablets duplos facilitaram o comportamento } \\
\text { comunicativo em parcerias adulto-criança do que os } \\
\text { tablets individuais. Essa estratégia, atrelada ao uso da } \\
\text { tecnologia, promoveu atividades colaborativas visando o } \\
\text { desenvolvimento sócio-cognitivo das crianças nesse } \\
\text { processo. }\end{array}$ \\
\hline $\begin{array}{l}\text { Smartphone, } \\
\text { Laptop, } \\
\text { Tablet, } \\
\text { Videogames }\end{array}$ & 6 & $\begin{array}{l}\text { O monitoramento dos pais foi considerado um } \\
\text { moderador estatisticamente significativo da relação } \\
\text { entre a percepção dos pais sobre o uso de dispositivos } \\
\text { móveis e sobre a competência social das crianças. A }\end{array}$ \\
\hline
\end{tabular}

Interfaces da Educ., Paranaíba, v.11, n.32, p. 159 - 187, 2020 


\begin{tabular}{|c|c|c|}
\hline $\begin{array}{l}\text { de console e } \\
\text { portátil, Mp3 }\end{array}$ & & $\begin{array}{l}\text { mudança social positiva deste estudo pode incluir } \\
\text { aliviar o equivoco de que a interação digital impediu o } \\
\text { desenvolvimento social, promovendo o papel dos pais } \\
\text { em criar crianças socialmente competentes na era } \\
\text { digital e defendendo uma estratégia de monitoramento } \\
\text { parental mais colaborativo. }\end{array}$ \\
\hline $\begin{array}{c}\text { Smartphone e } \\
\text { Tablets }\end{array}$ & 7 & $\begin{array}{l}\text { Buscando as possiveis mudanças no ato de tocar } \\
\text { permeadas por dispositivos móveis, identificou-se a } \\
\text { plasticidade dos dispositivos móveis como um elemento } \\
\text { importante, pois a tela se modifica e se adapta de } \\
\text { acordo com o toque da criança, despertando seu } \\
\text { interesse em interagir com esses dispositivos. A } \\
\text { possibilidade de que os dispositivos móveis funcionem } \\
\text { como ativadores da ZDP, convertendo o potencial real; e } \\
\text { a interação e a mediação também se destacaram, pois } \\
\text { quando a criança interage, aumenta a possibilidade de } \\
\text { que ela construa novos elementos cognitivos. }\end{array}$ \\
\hline $\begin{array}{l}\text { Tecnologias } \\
\text { digitais e } \\
\text { analógicas }\end{array}$ & 8 & $\begin{array}{l}\text { Os resultados revelam que as crianças brincavam em } \\
\text { grupos, exibindo uma infinidade de comportamentos } \\
\text { sociais e interações e variados graus de participação } \\
\text { social, e assumiram vários papéis de status social e } \\
\text { posições tecnológicas. Estes comportamentos faziam } \\
\text { parte de um sistema de reprodução digital, que por sua } \\
\text { vez foi influenciado pelo sistema pré-escolar, que } \\
\text { compreende crianças e praticantes como agentes ativos, } \\
\text { com capacidades tecnológicas, rotinas e práticas } \\
\text { culturais do cenário da primeira infância. Em última } \\
\text { análise, as experiências sociais das crianças durante o } \\
\text { jogo digital não podem ser determinadas por nenhum }\end{array}$ \\
\hline
\end{tabular}




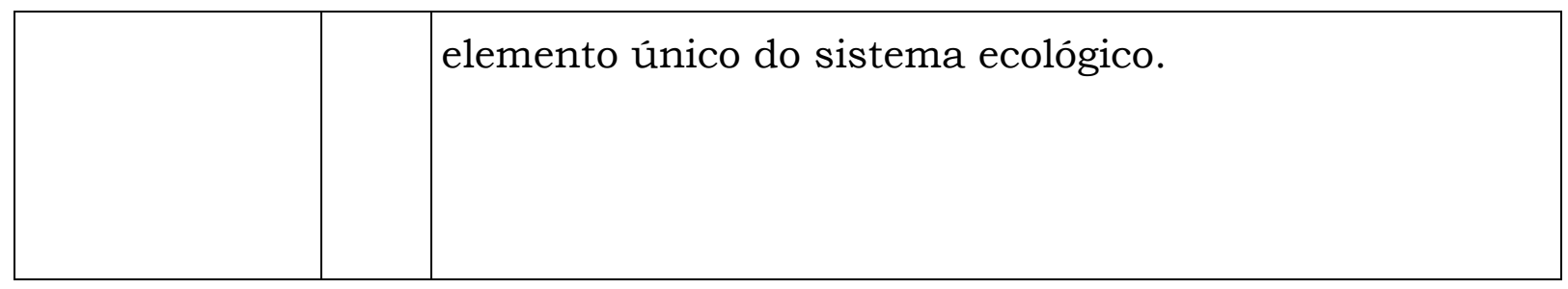

Fonte: Elaboração da autora, 2018.

Conforme apresentado no quadro anterior, dos oito trabalhos encontrados cinco deles tiveram como enfoque a pesquisa no uso de tablets pelas crianças na análise sobre a interação social. Com a análise dos resultados dessas pesquisas foi constatado que o uso da tecnologia digital pode motivar a interação a social (SAKR, KUCIRKOVA, 2017) e uma delas identificou que pode também influenciar no desenvolvimento de comportamentos comunicativos em crianças com autismo (HOLT; YUILL, 2017).

No entanto, quando o tablet ainda é novidade observam-se evidências, em uma das pesquisas, de que a criança acaba ficando mais imersa no dispositivo em detrimento da interação social (VOKATIS, 2014). Assim como, uma delas detalha que a tecnologia pode motivar tanto comportamento colaborativos como competitivos que dependerão de características individuais e culturais das crianças e das mediações presentes no contexto de uso (LAWRENCE, 2017).

As demais pesquisas, que analisavam outras tecnologias digitais móveis além do tablet, constataram que os seus usos geraram diferentes comportamento sociais, que podem contribuir no desenvolvimento da criança, pois criam novos elementos cognitivos a partir desse acesso (HOLT, YUILL, 2017; REYNOLDS-BLANKENSHIP, 2013; MENEGUZZO, 2014; ARNOTT, 2016). Assim como o monitoramento parental colaborativo (TOPPER, 2017) e o uso de determinados tipos de conteúdo que preveem o uso colaborativo (SAKR, KUCIRKOVA, 2017) também podem ser motivadores de interações sociais, demonstrando como equívoco se pensar que a tecnologia impede a interação. 
De modo geral, pode-se observar nos desfechos descritos que os trabalhos sugerem que as tecnologias digitais podem ser usadas mais para motivarem as relações sociais, ampliando as formas de comunicação e o desenvolvimento de competências digitais, do que resultarem em maior isolamento quando se tem o seu uso.

No entanto, existem alguns fatores que foram ponderados que podem motivar esse isolamento no uso, como aquele atrelado a novidade do acesso à tecnologia, que gera a imersão da criança durante nos primeiros contatos (SAKR, KUCIRKOVA, 2017), como os conteúdos que são utilizados que podem promover tanto comportamentos colaborativos como competitivos (LAWRENCE, 2017), e ainda as formas de mediação parental durante o acesso, que contribuem para que os usos motivem interações sociais, quando realizada de forma colaborativa com a criança (TOPPER, 2017).

\section{Discussão}

A partir dos resultados apresentados e pensando diretamente na interferência das tecnologias digitais nas relações sociais presenciais entre as crianças, será discutido o que os pesquisadores constataram em relação a esse aspecto em suas pesquisas, apresentando possiveis variáveis que potencializam ou prejudicam essas relações quando o digital está em cena.

Ao observar as habilidades sociais que se constituem a partir das relações sociais estabelecidas, Sakr e Kucirkova (2017) buscaram analisar possibilidades de aproximar a relação entre pai e filho de três anos de idade no uso de diferentes tecnologias digitais e não-digitais como colagem, giz de cera, aplicativo de tablet e o software Tuxpaint no laptop. A partir disso, as autoras identificaram que não foi o diferente tipo de tecnologia (digital ou não) que interferiu no nível de interação entre o pai e a criança, mas foram as potencialidades dos recursos, as possibilidades e os modos de uso percebidos por ambos que determinou uma maior ou menor interação social.

Nesse caso, a tecnologia foi utilizada como um potencializador da relação social entre pai e filho por meio de uma estratégia de produção de 
arte colaborativa, diferente da pesquisa de Uhls et al. (2014) que excluiu o uso da tecnologia para verificar a melhora na qualidade da comunicação não-verbal entre adolescentes. Desse modo, é possivel visualizar os contrapontos de se pensar no uso da tecnologia e do seu impacto sobre a relação social que é condizente ao objetivo que se propõe em cada acesso e atividades desempenhadas no uso das tecnologias digitais.

Além desses fatores que influenciam no grau de interação, na pesquisa de Vokatis (2014) que analisou a natureza das interações entre uma criança de cinco anos e seus pais durante o uso de aplicativos no tablet, a autora constatou que quando a ferramenta é adquirida recentemente as interações dialógicas entre pais e a criança são ainda mais dificeis de serem realizadas devido à novidade que acarreta maior imersão da criança na interação com a tecnologia.

Nesse sentido, para além da própria tecnologia e do objetivo que se propõe com ela, existem outros fatores como o nivel de familiaridade com o dispositivo, o domínio das ferramentas e o ambiente do contexto de apropriação que vão refletir sobre como as relações se dão durante esse acesso (BRONFENBRENNER, 1994).

Numa outra pesquisa que buscou analisar em que medida o uso de tecnologia digital afetaria a competência social das crianças de 5 a 12 anos a partir da percepção e monitoramento de 401 famílias, Topper (2017) identificou que a competência social se desenvolve de forma similar tanto no mundo digital como na realidade cotidiana, e que os pais desempenham um papel importante no monitoramento e regulação do uso pelas crianças, desde que essa mediação ocorra a partir de uma relação de colaboração entre ambos.

Para verificar isso o autor se pautou na teoria Vygotskyana (2007) e no Modelo de Schramm de Comunicação (1971). Na perspectiva de Vygotsky (2007), Topper (2017) verificou que o uso de tecnologia digital pelas crianças não interfere no desenvolvimento da competência social delas, pois elas lidam com artefatos que fazem parte do seu meio, e que com o apoio da mediação dos adultos a criança tende a desenvolver a Zona de Interfaces da Educ., Paranaíba, v.11, n.32, p. 159 - 187, 2020 
Desenvolvimento Proximal (ZPD). Apesar disso, o autor indica a necessidade de um estudo longitudinal para aprofundar a análise e os resultados descritos.

Topper (2017) cita ainda a teoria de Schramm (1971), que considera que quando a comunicação ocorre mediada por um meio, tanto face a face como por texto, imagem etc, para reforçar que é necessário haver um feedback constante entre remetentes e receptores. Isso garante a compreensão da mensagem transmitida, considerando as especificidades da interação face a face, como os sinais faciais, gestos e linguagem corporal que acabam se perdendo dependendo do tipo de comunicação digital estabelecida (DRUSSEL, 2012).

A partir dessa pesquisa Topper (2017) concluiu que o monitoramento e mediação colaborativa das famílias influenciavam positivamente sobre a competência social das crianças em comparação aos métodos de monitoramento e supervisão dos pais que incluíam regras restritivas, pois acarretam dificuldades de desenvolvimento da autonomia, tomada de decisão e habilidades para resolver problemas. Além da proibição de uso limitar a abertura de discussões sobre experiências negativas nesses acessos, podendo fazer com que a criança mentisse sobre suas atividades digitais.

A partir das pesquisas citadas até o momento, percebe-se que quando a tecnologia digital é utilizada coletivamente ela pode gerar experiências que só são possiveis por meio da interação social durante esse acesso, o que demonstra o uso desses meios como motivadores das relações entre os indivíduos. No entanto, não se pode perder de vista que em alguns dos casos citados a própria tecnologia já era projetada para esse uso coletivo, o que contribuiu na qualidade das relações. Embora, em outros, apesar da tecnologia ser voltada para um uso pessoal, como o objetivo era de transformar a experiência de uso por meio de uma relação colaborativa, a partir das estratégias pedagógicas pensadas, o resultado da experiência também ganhou novos nuances a partir do uso coletivo. Como foi o caso da 
pesquisa realizada por Reynolds-Blankenship (2013) que integrou o tablet nas práticas de ensino e aprendizagem de um jardim de infância, o que possibilitou que as crianças estabelecessem suas interações sociais entre grupos controlando os conflitos nas relações e colaborando umas com as outras durante o uso livre de um jogo digital para apoiar a alfabetização digital e motivar interações.

Pautada sob o mesmo objetivo, Holt e Yuill (2017) propuseram a integração de tablets duplos, interligados por meio de um suporte, para facilitar o comportamento comunicativo na parceria entre adultos e crianças com a deficiência do transtorno de autismo, além de objetivar a interação entre elas mesmas, apesar de cada uma interagir em um dispositivo para realização da atividade. Essa estratégia, atrelada ao uso da tecnologia, promoveu atividades colaborativas visando o desenvolvimento sócio-cognitivo das crianças nesse processo.

Além da estratégia pedagógica, o conteúdo escolhido para ser utilizado na tecnologia com as crianças, pode motivar uma frequência de comportamentos, como foi o caso da análise feita por Creighton e Szymkowiak (2014) que verificaram que o uso de jogos cooperativos beneficia a interação social entre as crianças em sala de aula.

Esses resultados têm aproximações, inclusive, à investigação realizada por Knaul (2015) que tinha como objetivo analisar as contribuições da integração do tablet às práticas pedagógicas no Ensino Fundamental I. Nela, obteve-se como resultado que a tecnologia digital atrelada ao conteúdo utilizado, somados às estratégias pedagógicas planejadas para esse acesso, motivam práticas de colaboração diversas entre as crianças, desenvolvendo, inclusive, novos letramentos (KNAUL, 2015).

Muitos fatores estão implicados no uso da tecnologia digital, pois por vezes esse acesso é motivado por intencionalidades pedagógicas e em outras é proposto livremente como uma atividade ou brincadeira que faz parte do repertório da criança na contemporaneidade. No entanto, o que muda nesse processo quando esse acesso não é motivado por uma intencionalidade pedagógica que prevê atividades, mediações entre as crianças ou ações Interfaces da Educ., Paranaíba, v.11, n.32, p. 159 - 187, 2020 
objetivas para possibilitar o desenvolvimento de comportamentos ou aprendizados? Como ficam as interações sociais entre as crianças quando não há uma proposta específica planejada por trás desse uso?

De acordo como Arnott (2016), algumas tecnologias possibilitavam o uso compartilhado, gerando colaboração e interação social entre as crianças ao permitir que ambas controlassem a tecnologia. Percebeu-se, ainda, que as crianças incorporaram diferentes funções como de líder, membro em interação e membro não interagente em suas brincadeiras. Por fim, salientou-se que um único fator não é determinante para gerar um comportamento, pois existem diferentes variáveis relacionadas ao espaço fisico, social e cultural que interferem nas relações sociais entre as crianças ao brincar (ARNOTT, 2016).

As próprias características individuais da criança, a sua cultura, a rotina de sala de aula e a mediação dos adultos em sua volta, além das próprias especificidades da tecnologia digital, interferem na forma das crianças se comportarem na brincadeira com o uso dela. Inclusive, esses fatores contribuem para que a criança manifeste comportamentos sociais tanto competitivos como também colaborativos durante essa interação, conforme verificou Lawrence (2017) numa pesquisa realizada com crianças com o uso de diferentes aplicativos de tarefas, exploração, construção e simulação por meio do tablet na pré-escola.

Alguns pesquisadores como Brooker e Siraj-Blatchford (2002) abordam que as crianças menores não distinguem o uso de brinquedos físicos dos digitais, assim como fazem os adultos, tratando-os como semelhante na manipulação de símbolos e imagens como uma nova forma de brincadeira simbólica. Assim, ao considerar que a atividade cognitiva não se limita ao uso de instrumentos e signos, influenciando sobre o comportamento humano (VIGOTSKY, 2007), cabe compreender melhor o modo como essas tecnologias constituem-se como instrumentos e toda a diversidade de signos que são combinados de maneira interativa e imersiva nas tecnologias digitais. Supõe-se que se podem ter diferentes 
comportamentos dependendo do tipo de instrumento manipulado e atividade realizada, por isso a importância de ater-se a influencia da interação com essas tecnologias, especialmente, quando realizada por crianças.

Em contrapartida, numa outra pesquisa que verificou quais mudanças que a inserção de tecnologias móveis, smartphones e tablets, promovem no brincar de crianças na faixa etária de três a quatro anos no contexto escolar, percebeu-se que a plasticidade embutida nas suas diferentes funções, como simulações, opções de telas e links, geram uma dinamicidade na interação que não é estática, como ocorre com um brinquedo físico. Isso demonstrou um aumento na possibilidade da criança construir novos elementos cognitivos a partir desse acesso, ativando potenciais da ZDP (VYGOTSKY, 2007), por meio da interação e mediação necessárias nessa atividade (MENEGUZZO, 2014).

De acordo com as pesquisas citadas, podem-se perceber proposições de uso da tecnologia para promover e posteriormente analisar as relações entre usuários quando o digital está em cena. Ao mesmo tempo em que é possivel identificar variáveis e fatores que influenciam nos usos que são feitos dos dispositivos pelas crianças, geralmente propondo uma relação de causa e efeito. Entretanto, além do processo em si, durante as interações sociais, existem diferentes fatores que podem influir em sentidos e modos de pensar, sentir e agir da criança advinda do seu contexto de vida em meio às situações que são vivenciadas e constantemente ressignificadas.

\section{Considerações finais}

Os trabalhos selecionados e analisados a partir da revisão sistemáticas nas bases de dados contribuíram para caracterização das pesquisas e seus resultados em relação às interações sociais durante o uso de tecnologias digitais na infância. Isso contribuiu para verificar as diferentes possibilidades de análise de um fenômeno a partir das teorias escolhidas para tecer a discussão da pesquisa empírica. Em contrapartida, o trabalho de revisão sistemática ampliou o desafio para definição de teorias que dessem conta do fenômeno encontrado, sem cair em determinismos Interfaces da Educ., Paranaíba, v.11, n.32, p. 159 - 187, 2020 
epistemológicos que muitas vezes acabam por reduzir ou enquadrar aspectos complexos de definir que são encontrados no campo.

De modo geral, os estudos analisados evidenciam que o uso de tecnologias digitais na infância em atividades, especialmente quando mediadas por pais ou professores, podem contribuir com a ampliação das habilidades sociais das crianças, pois inclui novas alternativas e modo de comunicação, inclusive, preparando melhor as crianças para atuarem um contexto contemporâneo que pressupõe o desenvolvimento de competências digitais.

Ao mesmo tempo, observa-se que o tipo de tecnologias e o ambiente em que a crianças fazem uso delas pode influenciar sobre as interações sociais. Ambientes como a escola em que há mediadores presentes e a possibilidade constante de interação social contribuem para o não isolamento e mesmo permitem mobilizar o diálogo e a colaboração no uso das tecnologias.

Nesse sentido, ao pensar nos usos e nas formas como as tecnologias digitais são inseridas em contextos educacionais, é importante se pensar sobre o tipo de conteúdo em relação ao objetivo fim, o modo de uso desse conteúdo, se individual ou coletivo, os tempos e as mediações que serão feitas a partir desse acesso, para que ao invés das tecnologias digitais causarem um isolamento entre as crianças em detrimento do seu uso, elas possibilitarem a ampliação de experiências entre elas e a colaboração, na realização de uma atividade comum.

\section{Referências}

ALMEIDA, Maria Elizabeth Bianconcini de; SILVA, Maria da Graça Moreira. Currículo, Tecnologia e Cultura Digital: Espaços e Tempos de Web Currículo. Revista e-curriiulum, v. 7, n. 1, p. 1-19, 2011.

ARNOTT, Lorna. An ecological exploration of young children's digital play: framing children's social experiences with technologies in early childhood. Early Years, v. 36, n. 3, p. 271-288, 2016. 
ASTINGTON, J. W. Sometimes necessary, never sufficient: False-belief understanding and social competence. In: REPACHOLI, B.; SLAUGHTER, V. (Org.). Individual differences in theory of mind: Implications for typical and atypical development. New York, NY: Psychology Press, 2003. p. 13-38.

BLANDON, A. Y., CALKINS, S. D., GRIMM, K. J., KEANE, S. P., \& O’BRIEN, M. Testing a developmental cascade model of emotional and social competence and early peer acceptance. Development and Psychopathology, v. 22, p. 737-748, 2010.

BOSACKI, S., ASTINGTON, J. W. Theory of mind in preadolescence: Relations between social understanding and social competence. Social Development, v. 8, n.2, p. 237-255, 1999.

BRONFENBRENNER, Urie. Ecological models of human development. Readings on the development of children, v. 2, n. 1, p. 37-43, 1994.

BROOKER, Liz; SIRAJ-BLATCHFORD, John. 'Click on Miaow!': how children of three and four years experience the nursery computer. Contemporary Issues in Early Childhood, v. 3, n. 2, p. 251-273, 2002.

BROWN, Ari; SHIFRIN, Donald L.; HILL, David L. Beyond 'turn it off': How to advise families on media use. AAP News, v. 36, n. 10, p. 54-54, 2015.

COLL, C; MONEREO, C. Psicologia da educação virtual: aprender e ensinar com as tecnologias da informação e comunicação. Porto Alegre: Artmed, 2010.

CREIGHTON, Susan; SZYMKOWIAK, Andrea. The effects of cooperative and competitive games on classroom interaction frequencies. Procedia-Social and Behavioral Sciences, v. 140, p. 155-163, 2014.

DRUSSELL, J. Social Networking and Interpersonal Communication and Conflict Resolution Skills among College Freshmen. St. Catherine University, 2012. Disponivel em: <http://sophia.stkate.edu/msw_papers/21>. Acesso em: Jun 2018.

EY, L.A.; CUPIT, C. G. Exploring young children's understanding of risks associated with Internet usage and their concepts of management strategies. Journal of Early Childhood Research, v. 9, n. 1, 2011. 
GIEDD, J. N. The digital revolution and adolescent brain evolution. Journal of Adolescent Health, v.51, p. 101-105, 2012.

HOLT, S., YUILL, N. Tablets for two: How dual tablets can facilitate otherawareness and communication in learning disabled children with autism. International Journal of Child-Computer Interaction, v. 11, p. 72-82, 2017.

KNAPP, M. L, HALL, J. A. Nonverbal communication in human interaction (Seventh.). Boston, MA: Wadsworth Cengage Learning, 2010.

KNAUL, Ana Paula. Novos letramentos na escola: uma análise da integração do tablet às práticas pedagógicas no Ensino Fundamental. Dissertação de Mestrado do Programa de Pós-Graduação em Educação da Universidade Federal de Santa Catarina. 2015.

LAWRENCE, Sandra M. Preschool children and iPads: Observations of social interactions during digital play. Early Education and Development, v. 29, n. 2, p. 207-228, 2018.

LIVINGSTONE, S. et. al. In their own words: What bothers children online? London, UK: EU Kids Online. 2013.

LIVINGSTONE, S. EU Kids Online: Findings, methods, recommendations. 2014. Disponivel em: http://1sedesignunit.com/EUKidsOnline/. Acesso em: 02 Jun 2018.

MENEGUZZO, Lorivane Aparecida. O brincar na educação infantil: a influência das tecnologias digitais móveis no contexto da brincadeira. Dissertação de Mestrado do Programa de Pós Graduação em Educação da Universidade de Caxias Do Sul: Caxias do Sul, 2014.

OKOLI, C. A guide to conducting a standalone systematic literature review. Communications of the Association for Information Systems, v.37, p. 1-33, 2015.

REYNOLDS-BLANKENSHIP, Tara Lynn. An action research study investigating children's use of an iPad during free play in a kindergarten classroom: An exploration of teaching pedagogy and children's learning, social 
interactions, and digital literacy. Tese de Doutorado. Texas Woman s University, 2013.

ROCCA, Cristina C. A., POLISEL, Adriana F., MATTOS, Karen M. G., SILVA, Maria Cecília F. Habilidade sociais. In: MALLOY-DINIZ, Leandro F. et al. Avaliação neuropsicológica. Porto Alegre: Artmed, 2010.

RUBIN, K. H., ROSE-KRASNOR, L. Interpersonal problem solving and social competence in children. In V. B. Van Hasselt \& M. Hersen (Eds.), Perspectives in developmental psychology. Handbook of social development: A lifespan perspective (pp. 283-323). New York, NY, US: Plenum Press. 1992.

SAKR, Mona; KUCIRKOVA, Natalia. Parent-child moments of meeting in artmaking with collage, iPad, Tuxpaint and crayons. International Journal of Education \& the Arts, v. 18, n. 2, 2017.

SAMPAIO; MANCINI. Estudos de revisão sistemática: um guia para síntese criteriosa da evidência científica. Rev. bras. fisioter, São Carlos, v. 11, n. 1, p. 83-89, 2007.

SANTISARUN, P.; BOONKRONG, S. Social network monitoring application for parents with children under thirteen. In: Knowledge and Smart Technology (KST), 7th International Conference on, 2015. p. 75-80.

SCHRAMM, W. The nature of communication between humans. In W.Schramm \& D.F. Roberts (Eds.). The process and effects of mass communication. Urbana: University of Illinois Press, 1971. p. 3-53.

TOPPER, Christin. Parental Perception of Mobile Device Usage in Children and Social Competency. Tese de Doutorado. EUA: Walden University. 2017.

TSCHOLL, Michael; LINDGREN, Robb. Empowering digital interactions with real world conversation. TechTrends, v. 58, n. 1, p. 56-63, 2014.

UHLS, Y. T., MICHIKYAN, M., MORRIS, J., GARCIA, D., SMALL, G. W., ZGOUROU, E., GREENFIELD, P. M. Five days at outdoor education camp without screens improves preteen skills with nonverbal emotion cues. Computers in Human Behavior, v. 39, p. 387-392, 2014.

VOKATIS, Barbara M. Meaning Making with an iPad: A Case Study of One Interfaces da Educ., Paranaiba, v.11, n.32, p. 159 - 187, 2020 
Child's Engagement with iPad Applications within Her Family Activity System. Albany: State University of New York, 2014.

VYGOTSKY, Lev S. A formação social da mente: o desenvolvimento dos processos psicológicos superiores. $7^{\text {a }}$ ed. São Paulo: Martins Fontes, 2007. 